\title{
El modelo educativo en el contexto de la docencia universitaria
}

\author{
Carmen María Triminio Zavala, Juan Alberto Betanco Maradiaga, \\ Karen Elizabeth Velásquez Meza \\ Docentes de la Universidad Nacional Autónoma de Nicaragua, Managua \\ Facultad Regional Multidisciplinaria (FAREM-Estelí) \\ ctriminiozavala@gmail.com betancoja@yahoo.com meza0788@yahoo.es
}

\section{INTRODUCCIÓN}

La labor docente en la universidad, en su forma más extendida y tradicional se había enfocado en la transmisión del conocimiento que se desarrolla en las exposiciones dictadas por el docente y en la entrega de fotocopias de un libro proporcionado a los y las estudiantes siguiendo estrictamente el Programa de Asignatura ${ }^{1}$. Con esta metodología, quien más aprende es el docente, pues para preparar su clase el investiga buscando información en los libros y/o en la web, la analiza y luego la dicta; sin embargo lo que para nosotros cómo maestro es información para el estudiante simplemente son datos; por ello comparto lo que pregunta Van de Velde (2014), en su escrito docencia hoy... "Al preparar y desarrollar nuestro encuentros con estudiantes, ¿realmente estamos trabajando con información o solamente con datos encontrados desde alguna fuente? (p.1)

A partir del 2011 el proceso de enseñanza aprendizaje en la Universidad Nacional Autónoma de Nicaragua, Managua (UNAN-Managua) "tiene como base un modelo pedagógico que centra su atención en el estudiante, que asume su rol activo y participativo con una alta responsabilidad en el desarrollo de un aprendizaje autónomo y estratégico" (Modelo educativo, 2011, p. 23), el cual será posible con el acompañamiento del docente quien debe ser el primer sujeto de cambio, pues el que facilita el proceso enseñanza-aprendizaje.

Por lo antes mencionado, como docentes de la Facultad Regional Multidisciplinaria - Estelí (FAREM-Estelí) con este ensayo nos proponemos revisar sí nuestro quehacer docente, ¿es coherente con el 'modelo educativo' que ha adoptado nuestra institución educativa? Analizaremos desde cómo preparamos y organizamos la clase, qué estrategias de aprendizaje aplicamos en el proceso de enseñanza aprendizaje hasta la forma de evaluación de la asignatura.

\footnotetext{
${ }^{1}$ El programa de asignatura es un documento que contempla los objetivos generales y específicos, las unidades con sus temas y subtemas, algunas recomendaciones metodológicas y la bibliografía.
} 


\section{DESARROLLO}

A partir del 2013 que se implementó el nuevo modelo educativo en nuestra universidad, el cual está centrado en el estudiante que construye su propio conocimiento partiendo de sus experiencias, conocimientos e intereses previos que tienen los estudiantes para enlazarlos con el nuevo contenido, y lograr así un aprendizaje significativo mediante "la vinculación y aplicación de los contenidos en una variedad de situaciones y problemas de la vida real..." (Modelo educativo, 2011, p.25).

Según Sanmartí (2007) plantea que "La evaluación diagnóstica inicial tiene como objetivo fundamental analizar la situación de cada estudiante antes de iniciar un determinado proceso de enseñanza-aprendizaje,...” (p.33). Tomando en cuenta lo antes mencionado y lo que plantea el modelo educativo en la asignatura didáctica de la Física, se aplicó este tipo de evaluación, para conocer sí estudiantes de III año de la Carrera Física-Matemática saben sobre los modelos didácticos de la enseñanza de la Física ${ }^{2}$ al relacionar aspectos relacionados con ¿Para qué enseñar? ¿Qué enseñar? ¿Cómo enseñar? ¿Cómo evaluar?

La diagnosis permitió identificar que solo tres estudiantes (de 30 estudiantes que llevan está asignatura) son docentes de secundaria y por ende conocen los diferentes modelos educativos logrando identificarse en el modelo alternativo; los demás estudiantes conciben todavía el proceso de enseñanza tradicional por lo tanto el reto de facilitar la asignatura didáctica de la física fue mayor pero más enriquecedor.

La situación antes mencionada sirvió para facilitar dicha asignatura aplicando la siguiente frase "Dime y lo olvido, enséñame y lo recuerdo, involúcrame y lo aprendo"3. Es decir, involucre directamente a los estudiantes en el desarrollo de la clase aunque sean contenidos nuevos; por ejemplo la tercera unidad "La evaluación como regulación de los aprendizajes" ${ }^{4}$ se desarrolló con el análisis del libro 10 ideas clave Evaluar para aprender. Los y las estudiantes expusieron sus puntos de vista relacionándolo con las experiencias vividas en el desarrollo de las diferentes asignaturas que recibían en este semestre, sobre todo la idea clave "El error es útil para regular su aprendizaje" (Sanmartí, 2007, p.43).

\footnotetext{
${ }^{2}$ Adaptado de http://cmc.ihmc.us/cmc2006Papers/cmc2006-p215.pdf

${ }^{3}$ Frase célebre que dijo Benjamín Franklin (1706 - 1790), estadista y científico estadounidense.

${ }^{4}$ Programa de la asignatura didáctica de la Física
} 
La evaluación parcial $^{5}$ de esta clase no fue la tradicional prueba escrita. Por las características de la asignatura se realizó una evaluación colectiva en tríos que permitió lograr un proceso colectivo de discusión, análisis, de reconocimiento de aprendizajes y construcción de ideas plasmadas en un ensayo tomando como referencia la lectura realizada sobre el libro antes mencionado y la toma de apuntes del videoconferencias educativas "Evaluación centrada en los aprendizajes" presentado por Santos Guerra (2013) en la Escuela de Comercio Martín Zapata - Un Cuyo.

Para consolidar los contenidos analizados en esta asignatura, se orientó que los estudiantes elaboraran un cuento didáctico sobre: los aprendizajes alcanzados; las dificultades encontradas con las formas de cómo las superaron y lecciones aprendidas. Debía usar su imaginación y pictogramas ${ }^{6}$. Esto permitió aplicar la evaluación final no solo de forma cuantitativa, sino como plantea Sanmartí (2007) “por tanto, la evaluación final debería orientarse a ayudar a los alumnos a reconocer qué han aprendido y a tomar conciencia de las diferencias entre el punto de partida y el final” (p.35).

Cada asignatura que impartimos tiene su particularidad, por lo tanto otra de las experiencias de estrategias de aprendizaje aplicadas en la asignatura de Matemática General fue la aplicación de juegos matemáticos, que aunque tienen un carácter de algunos contenidos aritméticos, fundamentado en lo que plantea Guzman (1984)

¿Por qué no paliar la mortal seriedad de muchas de nuestras clases con una sonrisa? Si cada día ofreciésemos a nuestros alumnos, junto con el rollo cotidiano, un elemento de diversión, incluso aunque no tuviese nada que ver con el contenido de nuestra enseñanza, el conjunto de nuestra clase y de nuestras mismas relaciones personales con nuestros alumnos variarían favorablemente (p. 7).

En el estudio de ingeniería se toman en cuenta algunos aspectos como prácticas de laboratorio, elaboración de proyectos, resolución de ejercicios. El modelo educativo de UNAN-Managua (2011) contempla entre los aprendizajes estratégicos del currículo integral se encuentra el aprender hacer que desarrolla habilidades y destrezas. Cuando se plantea un proyecto, lo primero que se infiere es la idea de querer solucionar un problema o solventar necesidades evidentes. Plantear este tipo de tareas al estudiantado implica que estos se involucren en diversos procesos de construcción significativa de conocimientos.

\footnotetext{
${ }^{5}$ Examen correspondiente al 40\% de la calificación final de la asignatura.

${ }^{6}$ Dibujos simples y de fácil interpretación que representan acciones, lugares, instrumentos y permite transmitir, de este modo, una información también convencionalizada. (Documento de referencia, 2012, p.41)
} 
En II año de ingeniería en energías renovables en el desarrollo de la asignatura llamada Recursos energéticos renovables fuimos construyendo el proyecto de evaluar el potencial de los recursos energéticos de una comunidad determinada. Se evaluaron recursos solares, eólicos, hídricos y de biomasa adquiriendo los estudiantes destrezas para usar aparatos, recopilar datos, procesarlos estadísticamente y reconocer tecnologías energéticas.

Dentro del contexto de la ingeniería, debe remarcarse que la nueva tecnología no es otra manera de extender la practica educativa, si no que por si misma define nuestra cultura, sociedad y el mundo tan impredecible y cambiante. Su componente eminentemente práctico es hoy en día parte necesaria del rol que debe jugar la educación superior en cuanto a la preparación de los estudiantes para la cultura del futuro, dado que rápidamente se convierte en presente. En este sentido, la innovación por sí sola, llega a ser contenido en el currículo de la educación superior. (Light G, 2006, p. ).

Además, en la asignatura Electrónica a III de Ingeniería en energías renovables destacamos lo importante de utilizar tecnologías de información para crear competencias en el área de electrónica mediante el uso de software de simulación; en este caso para diseños y análisis del funcionamiento de circuitos electrónicos. Los estudiantes reconocen la relevancia para la aprehensión de los conocimientos teóricos el uso de simuladores que les permite analizar diversas variantes de circuitos hasta llegar al óptimo para proceder a su montaje.

Algo similar sucede en el caso de la asignatura de Cartografía y Sistemas de Información Geográficos (SIG) que se imparte a la carrera de Ingeniería Ambiental, en donde la parte más enriquecedora en el desarrollo de la asignatura es la complementación del conocimiento, abordando la parte teórica fundamental, con la resolución de problemas o estudios de casos in situ, donde los estudiantes tienen la oportunidad de desarrollar habilidades y destrezas en la aplicación de herramientas, utilización de instrumentos y software que permita la construcción y análisis de la información espacial, para la planificación y ordenamiento del territorio, desde los Sistemas de información Geográficos.

Todo esto aportando al fortalecimiento de los aprendizajes significativos personales y colectivos desde los contenidos de asignatura, así como el desarrollo de la conciencia crítica para el ejercicio de su carrera, así mismo ha dado la oportunidad para que los y las estudiantes autoevalúen el proceso de aprendizaje realizado a lo largo de estas disciplinas. 


\section{CONCLUSIONES}

Por todo lo antes mencionado podemos decir que nuestro quehacer educativo está de acorde al modelo educativo de la UNAN-Managua, en cuánto a preparar las asignaturas pensando en el estudiante, aplicando estrategias de aprendizaje que le permitan construir su propio aprendizaje de manera dinámica, práctica, mediante juegos y que sea significativo para ellos, sobre todo sí se les toma en cuenta al momento de ¿cómo evaluarlos? y ¿qué criterios se deben tomar para evaluarlos? Sin embargo, nos hace falta incorporar las tecnologías de la Información y la comunicación, sobre todo en la asignatura de matemática y física porque además de mejorar las prácticas pedagógicas en el aula también permite al estudiantado otros escenarios de aprendizaje.

Por lo tanto el reto es crear un blog donde se pueda compartir, intercambiar y retroalimentar los conocimientos y aprendizajes en conjunto con estudiantes de estas asignaturas.

\section{REFERENCIAS}

Guerra, S. (2 de Mayo de 2013). https://www.youtube.com. Recuperado el 18 de Octubre de 2014, de https://www.youtube.com/watch?v=Zkj-NhFSStk

Guzman, M. d. (s.f.). http://utenti.quipo.it/. Recuperado el 15 de Diciembre de 2014, de http://utenti.quipo.it/base5/introduz/guzmanjuegos.htm

Light G. and COX R. Learning and Teaching in Higher Education. SAGE Publications, Reprinted 2006, p.167

María Elena Blandón Dávila, C. M. (Junio de 2012). Capacitación dirigida a docentes de Asociación Familia Padre Fabretto. Estelí, Nicaragua.

Ricardo Chrobak, M. L. (2006). http://cmc.ihmc.us. Recuperado el 21 de Enero de 2014, de http://cmc.ihmc.us/cmc2006Papers/cmc2006-p215.pdf

Sanmartí, N. (2007). 10 ideas clave evaluar para aprender. Barcelona: GRAÓ.

Universidad Nacional Autónoma de Nicaragua UNAN-Managua. (6 de Mayo de 2011). Modelo

Educativo, Normativa y Metodología para la Planificación Curricular 2011. Managua, Nicaragua.

Velde, H. V. ( 18 de Agosto de 2014). Docencia hoy... ¿lo estamos haciendo bien? Estelí, Nicaragua. 